\title{
On nonstationarity and rippling of the quasiperpendicular zone of the Earth bow shock: Cluster observations
}

\author{
V. V. Lobzin ${ }^{1, *}$, V. V. Krasnoselskikh ${ }^{1}$, K. Musatenko ${ }^{1}$, and T. Dudok de Wit ${ }^{1}$ \\ ${ }^{1}$ LPCE/CNRS-Université d'Orléans, Orleans, France \\ *now at: the University of Sydney, Sydney, Australia
}

Received: 7 May 2008 - Revised: 21 July 2008 - Accepted: 11 August 2008 - Published: 23 September 2008

\begin{abstract}
A new method for remote sensing of the quasiperpendicular part of the bow shock surface is presented. The method is based on analysis of high frequency electric field fluctuations corresponding to Langmuir, upshifted, and downshifted oscillations in the electron foreshock. Langmuir waves usually have maximum intensity at the upstream boundary of this region. All these waves are generated by energetic electrons accelerated by quasiperpendicular zone of the shock front. Nonstationary behavior of the shock, in particular due to rippling, should result in modulation of energetic electron fluxes, thereby giving rise to variations of Langmuir waves intensity. For upshifted and downshifted oscillations, the variations of both intensity and central frequency can be observed. For the present study, WHISPER measurements of electric field spectra obtained aboard Cluster spacecraft are used to choose 48 crossings of the electron foreshock boundary with dominating Langmuir waves and to perform for the first time a statistical analysis of nonstationary behavior of quasiperpendicular zone of the Earth's bow shock. Analysis of hidden periodicities in plasma wave energy reveals shock front nonstationarity in the frequency range $0.33 f_{B i}<f<f_{B i}$, where $f_{B i}$ is the proton gyrofrequency upstream of the shock, and shows that the probability to observe such a nonstationarity increases with Mach number. The profiles observed aboard different spacecraft and the dominating frequencies of the periodicities are usually different. Hence nonstationarity and/or rippling seem to be rather irregular both in space and time rather than resembling a quasiregular wave propagating on the shock surface.
\end{abstract}

Keywords. Space plasma physics (Nonlinear phenomena; Shock waves; Waves and instabilities)

Correspondence to: V. V. Lobzin

(v.lobzin@physics.usyd.edu.au)

\section{Introduction}

The problem of shock front nonstationarity is of fundamental importance for collisionless shock physics because it is closely related to two main questions of this field of plasma physics, namely, (1) the dependence of shock front structure on upstream plasma parameters and shock velocity; (2) acceleration of particles up to high energies (Kennel et al., 1985). However, although this problem was raised in the very beginning of collisionless shock physics, after more than four decades of research it is far from being well understood, most probably due to formidable difficulties of the experimental and theoretical analysis. A brief summary of the state of art can be found in the papers by Krasnoselskikh et al. (2002) and Lobzin et al. (2007), as well as in recent reviews (Hellinger, 2003; Lembege et al., 2004; Bale et al., 2005).

Up to the present time the information about different aspects of shock front nonstationarity was obtained first of all in numerical modeling. The "macroscopic" nonstationary phenomena, which are related to low-frequency motions with large spatial scales, can be subdivided in two categories, i.e. one-dimensional and multidimensional nonstationarity. A phenomenon can be considered as intrinsically one-dimensional if it can be observed in a planar geometry. However, in a more realistic geometry this phenomenon can produce large-scale multidimensional patterns, but there can exist quite large regions of the shock front where the profiles of different physical parameters measured across the shock are very similar at the same time. One-dimensional phenomena like, for example, shock front nonstationarity and reformation were studied numerically for the first time by Biskamp and Welter (1972). On the other hand, there can exist essentially multi-dimensional effects like the so-called shock front rippling. In the present literature the term "rippling" is used to denote at least two phenomena that can be essentially different. In particular, Winske and Quest (1988)

Published by Copernicus Publications on behalf of the European Geosciences Union. 
as well as Lowe and Burgess (2003) assume that rippling means wave-like ion-scale features moving along the shock surface, while the features observed in the simulations performed by Thomas (1989) seem to be rather irregular. Probably it is worth to introduce for the latter phenomenon the term "wrinkling" while retaining the term "rippling" for the former one. There exists a possibility of combination of reformation with rippling or wrinkling, i.e. the reformation may proceed as a wave propagating on the shock surface or form a rather irregular pattern. A shock front instability found in the simulations by Burgess and Scholer (2007) can probably result in such kind of reformation. In the following we will focus first of all on rippling and wrinkling.

Shock front rippling was observed for the first time by Winske and Quest (1988) in two-dimensional hybrid simulations, where the wave-like disturbances were clearly seen both in the magnetic field and plasma density. These structures move along the shock surface with a speed of $0.3 V_{A}$ and have a characteristic scale of about $5 c / \omega_{p i}$ and a frequency of $0.4 \omega_{B i}$, where $V_{A}$ is the Alfvén velocity, $c$ is the speed of light, $\omega_{p i}$ is the proton plasma frequency, and $\omega_{B i}$ is the proton gyrofrequency (unless otherwise stated, all characteristic velocities and scales are calculated with upstream plasma parameters). Disturbances with similar scales were also found by Thomas (1989) in two- and three-dimensional simulations. He focused on dimensionality effects on amplitudes of disturbances and did not analyze their motion. It is worth noting, however, that disturbances observed by Thomas (1989) seem to be more chaotic than those seen by Winske and Quest (1988), and the randomness is considerably higher in the three-dimensional case than in the twodimensional one.

Later Burgess (2006) performed a simulation of rippling to analyze the problems of interpretation of multipoint observations. However, for such a complicated problem a single case study cannot be considered as definite evidence favoring shock front rippling. In addition, a lot of questions still remain open. In particular, can the wrinkling and/or rippling be considered as a typical feature and how do they depend on plasma and shock parameters?

Up to now there are only a few experimental studies concerning shock front nonstationarity both in space and laboratory plasmas.

The first unambiguous evidence of the shock wave nonstationarity was obtained by Morse et al. (1972) in laboratory experiments with a plasma-wind-tunnel device. They revealed that the high-Mach-number shock wave oscillates with a frequency comparable to the upstream ion gyrofrequency.

Vaisberg et al. (1984) reported low frequency oscillations of the ion flux in the Earth's bow shock. Bagenal et al. (1987) observed a similar phenomenon in the Uranian bow shock. The manifestation of the shock front nonstationarity in the ion distribution function was also discussed by Krasnoselskikh et al. (1990).
Galeev et al. (1988a, b) presented the results of analysis of the magnetic field measurements performed onboard Prognoz- 8 and Prognoz-10 for several crossings of the Earth's bow shock and discussed the role of nonlinear whistler waves in the shock front nonstationarity. Later Krasnosel'skikh et al. (1991) and Balikhin et al. (1997) analyzed observations of Prognoz-10 and AMPTE spacecraft and confirmed that the main low frequency oscillations found in the shock front are whistlers. The amplitude of these waves is large and cannot be explained as a result of an instability driven by reflected ions, rather, they can be considered as an intrinsic element of the shock front structure generated by the processes of the nonlinear macrodynamic of the front (Galeev et al., 1988a; Krasnosel'skikh et al., 1991; Walker et al., 1999a).

Walker et al. (1999b) presented observations of a quasiperpendicular shock encountered by APMTE-UKS and AMPTE-IRM with quite different profiles for the two spacecraft and interpreted these observations as a result of shock front nonsationarity. With the use of Cluster data, Horbury et al. (2001) found examples of some aspects of shock nonstationarity, where the amplitude of magnetic field fluctuations attains $\sim 10 \mathrm{nT}$, making profiles considerably different for different spacecraft. Horbury et al. (2001) argue that these fluctuations stop before the ramp and do not appear to disrupt the shock structure; on the other hand, they don't reject the possibility that the fluctuations may be signatures of the unsteady shock reformation. The first convincing experimental evidence in favor of shock front reformation was presented by Lobzin et al. (2007).

Moullard et al. (2006) were the first who probably observed another aspect of shock front nonstationarity - rippling. They analyzed a single event when during $\sim 1 \mathrm{~h}$ time interval Cluster spacecraft "touched" the bow shock and than crossed it twice during $\sim 10 \mathrm{~min}$. This shock is almost perpendicular, high-beta, and high-Mach-number, with the fast mode Mach number $M_{f}=11$. Moullard et al. (2006) argue that the observed oscillations of the magnetic field and plasma density within the front can be interpreted as a wave moving along the shock surface. The velocity of this wave seems to make an acute angle $\left(<40^{\circ}\right)$ with the upstream magnetic field and varies within the range from 2 to $4 V_{A d}(80-$ $160 \mathrm{~km} / \mathrm{s}$ ), where $V_{A d}$ is the downstream Alfvén velocity. The wavelength estimated by Moullard et al. (2006) is 1000$2000 \mathrm{~km}$.

Up to the present time, the information concerning different aspects of shock front nonstationarity was obtained first of all in numerical modeling, which allows one to follow the temporal evolution and spatial structure of the shock wave with an arbitrary resolution.

A serious limitation of space experiments is that the spatial resolution is always limited to spacecraft separation, which could be too large for deducing any information about the fields in between. For example, the spacecraft separation varies in the range $100-10000 \mathrm{~km}$ for Cluster mission, while 
the thickness of a typical quasiperpendicular shock is about $600 \mathrm{~km}$. However, when spacecraft are in the electron foreshock, there exists an indirect method for remote sensing of the bow shock surface during relatively long time intervals (see Lobzin et al., 2003, and the details in the following). This method is based on analysis of high-frequency electric field fluctuations corresponding to Langmuir, upshifted, and downshifted waves. These waves are generated by superthermal electrons that were reflected from the bow shock and propagate in the sunward direction along the magnetic field lines (e.g. Filbert and Kellogg, 1979; Lacombe et al., 1985; Lobzin et al., 2005, and references therein). The deformation of the shock front should lead to significant changes of these fluxes, which in their turn result in changes of observed electric field spectra.

In the present paper we use the above-mentioned method for remote shock front sensing and present the results of statistical study of hidden periodicities in the measured Langmuir wave energies. The main aim of the paper is to study spatio-temporal characteristics of shock front nonstationarity and the search of experimental evidence favoring shock front rippling and/or wrinkling. This aim is accomplished by describing the data selection and analysis procedure (Sect. 2), interpreting and discussing the results (Sect. 3), and then giving the conclusions (Sect. 4).

\section{Data selection and analysis procedure}

The direct observation of shock front rippling and/or wrinkling onboard spacecraft is rather difficult, because a typical time of bow shock crossing is a few ion gyroperiods and is comparable or even less than the expected characteristic time scale of rippling, and the coherence length for this process can be less than the distance between the satellites, thereby making useless the multi-spacecraft methods for separations of spatial and temporal variations. To find convincing experimental evidence of shock front rippling or wrinkling, it is desirable to follow the shock evolution during relatively long time intervals, much longer than the ion gyroperiod. Fortunately, when spacecraft are in the electron foreshock, there exists a possibility for remote sensing of the quasiperpendicular region of bow shock surface during relatively long time intervals (Lobzin et al., 2003). To this end, one can analyze high-frequency electric field fluctuations corresponding to Langmuir, upshifted, and downshifted waves. These waves are generated by suprathermal electron fluxes, which are reflected from the bow shock front and move approximately along the magnetic field lines (see, e.g. Filbert and Kellogg, 1979; Lacombe et al., 1985; Lobzin et al., 2005, and references therein). Any deformation of the shock front should lead to significant changes of these fluxes, which in their turn result in changes of observed electric field spectra.

Indeed, from the theory of energization of electrons by shock waves it follows that the key parameters of the dis- tribution function of reflected electrons depend strongly on the angle between the shock normal and the upstream magnetic field, $\theta_{B n}$ (Leroy and Mangeney, 1984; Wu, 1984). In particular, for typical parameters of the Earth bow shock, the average energy per electron increases from $\sim 4 T_{e}$ to $\sim 50 T_{e}$ within relatively narrow range of angles, from $83^{\circ}$ to $88^{\circ}$, while the relative number density drops from $11 \%$ to $0.3 \%$ and becomes negligible for angles larger than $89^{\circ}$ (Leroy and Mangeney, 1984). Thus energetic electrons that are responsible for the generation of intense plasma waves in the electron foreshock come from a relatively small region of the bow shock, near the point of tangency of the solar wind magnetic field. Any nonstationarity of the shock in this region will result in nonstationary behavior of Langmuir waves in the vicinity of foreshock boundary. In particular, even if the shock nonstationarity is moderate, a strong modulation of Langmuir wave intensity is expected to be observed due to sharp dependence of electron beam parameters on the $\theta_{B n}$. On the other hand, Lowe and Burgess (2003) argue that the amplitude of rippling increases as $\theta_{B n}$ approaches $90^{\circ}$, thereby favoring the modulation of electron fluxes and Langmuir waves. Numerical studies of the electron energization in the time-dependent electromagnetic fields typical for the Earth's bow shock show that the modulation can be very strong, i.e. upstream of the re-forming shock energetic electron bursts can be formed cyclically rather than resemble a continuous electron beam (Yuan et al., 2007). It is also worth noting that, in accordance both with the theory and simulations, shock front nonstationarity not related to rippling and/or wrinkling should be observed both for perpendicular and quasiperpendicular shocks in the wide range of $\theta_{B n}$ provided that Mach number is high enough (e.g. Krasnoselskikh et al., 2002; Hellinger, 2003; Lembege et al., 2004, and references therein).

Thus, although spacecraft rarely cross the perpendicular zone of the Earth's bow shock, crossing of the electron foreshock boundary occurs much more frequently and intense Langmuir waves in the vicinity of this boundary do provide a convenient tool for remote sensing of this portion of the shock surface. The most favorable situation takes place when the solar wind is quiet and the frequencies of downshifted oscillations are well below the plasma frequency because in this case one can study both the wave intensities, separately for each mode, and variations of their frequencies.

The experimental data considered in this study were obtained aboard Cluster spacecraft by the WHISPER instrument between January and early May 2001. During this time period the four Cluster spacecraft crossed the Earth's bow shock many times and made numerous observations of the foreshock wave activity.

The WHISPER instrument was described by Décréau et al. (1997). This instrument is an element of Wave Experiment Consortium (WEC) presented by Pedersen et al. (1997). In the passive mode of operation WHISPER provides the calculated onboard electric field spectra of natural emissions in 
Table 1. The list of the events and their duration.

\begin{tabular}{|c|c|c|c|}
\hline No & Date & Time, UT (h:m:s) & $\begin{array}{c}\text { Duration for } \\
\text { Sc1, min }\end{array}$ \\
\hline 1 & 3 Jan 2001 & $01: 22: 14-01: 24: 06$ & 1.86 \\
\hline 2 & 3 Jan 2001 & $03: 40: 21-03: 43: 33$ & 3.19 \\
\hline 3 & 5 Jan 2001 & $22: 00: 04-22: 01: 58$ & 1.90 \\
\hline 4 & 6 Jan 2001 & $00: 42: 23-00: 46: 13$ & 3.83 \\
\hline 5 & 7 Jan 2001 & $23: 37: 45-23: 40: 37$ & 2.86 \\
\hline 6 & 15 Jan 2001 & $03: 19: 30-03: 22: 01$ & 2.51 \\
\hline 7 & 17 Jan 2001 & 08:19:01-08:21:49 & 2.79 \\
\hline 8 & 20 Jan 2001 & $00: 31: 32-00: 33: 34$ & 2.02 \\
\hline 9 & 20 Jan 2001 & $00: 39: 14-00: 42: 06$ & 2.76 \\
\hline 10 & 20 Jan 2001 & $18: 53: 29-18: 56: 57$ & 3.45 \\
\hline 11 & 29 Jan 2001 & $02: 42: 10-02: 46: 10$ & 3.92 \\
\hline 12 & 31 Jan 2001 & $12: 21: 23-12: 22: 48$ & 1.41 \\
\hline 13 & 31 Jan 2001 & $12: 31: 35-12: 34: 12$ & 2.61 \\
\hline 14 & 31 Jan 2001 & $15: 44: 39-15: 46: 42$ & 2.05 \\
\hline 15 & 31 Jan 2001 & $17: 12: 09-17: 15: 33$ & 3.40 \\
\hline 16 & 3 Feb 2001 & $13: 29: 47-13: 30: 58$ & 1.17 \\
\hline 17 & 10 Feb 2001 & $03: 30: 28-03: 33: 28$ & 2.98 \\
\hline 18 & 12 Feb 2001 & $08: 18: 57-08: 21: 48$ & 2.84 \\
\hline 19 & 13 Feb 2001 & $01: 13: 52-01: 16: 54$ & 3.01 \\
\hline 20 & 14 Feb 2001 & $17: 54: 51-17: 57: 43$ & 2.86 \\
\hline 21 & 21 Feb 2001 & 19:06:18-19:09:03 & 2.74 \\
\hline 22 & 21 Feb 2001 & $21: 35: 37-21: 37: 33$ & 1.93 \\
\hline 23 & 22 Feb 2001 & $23: 33: 26-23: 36: 48$ & 3.36 \\
\hline 24 & 23 Feb 2001 & $00: 38: 12-00: 41: 36$ & 3.39 \\
\hline 25 & $28 \mathrm{Feb} 2001$ & $21: 30: 06-21: 34: 58$ & 4.84 \\
\hline 26 & 7 Mar 2001 & 01:06:02-01:08:52 & 2.83 \\
\hline 27 & 13 Mar 2001 & $10: 13: 19-10: 17: 28$ & 4.15 \\
\hline 28 & 17 Mar 2001 & 19:29:40-19:31:09 & 1.44 \\
\hline 29 & 17 Mar 2001 & $22: 15: 04-22: 17: 12$ & 2.13 \\
\hline 30 & 18 Mar 2001 & $01: 41: 57-01: 45: 32$ & 3.58 \\
\hline 31 & 19 Mar 2001 & 19:09:13-19:10:59 & 1.75 \\
\hline 32 & 20 Mar 2001 & $00: 23: 58-00: 25: 40$ & 1.69 \\
\hline 33 & 20 Mar 2001 & $05: 20: 49-05: 23: 36$ & 2.78 \\
\hline 34 & $22 \operatorname{Mar} 2001$ & 09:15:08-09:18:00 & 2.85 \\
\hline 35 & $24 \operatorname{Mar} 2001$ & $14: 35: 57-14: 37: 46$ & 1.82 \\
\hline 36 & 24 Mar 2001 & $19: 11: 48-19: 13: 46$ & 1.96 \\
\hline 37 & 27 Mar 2001 & $08: 24: 12-08: 26: 23$ & 2.18 \\
\hline 38 & $28 \operatorname{Mar} 2001$ & $05: 24: 08-05: 26: 00$ & 1.85 \\
\hline 39 & 29 Mar 2001 & $08: 25: 49-08: 27: 35$ & 1.76 \\
\hline 40 & 2 Apr 2001 & $02: 12: 14-02: 15: 13$ & 2.98 \\
\hline 41 & 2 Apr 2001 & $03: 16: 05-03: 18: 30$ & 2.39 \\
\hline 42 & 5 Apr 2001 & $16: 34: 38-16: 37: 30$ & 2.86 \\
\hline 43 & 5 Apr 2001 & $21: 36: 29-21: 38: 38$ & 2.15 \\
\hline 44 & 10 Apr 2001 & $14: 52: 16-14: 54: 37$ & 2.34 \\
\hline 45 & 15 Apr 2001 & $18: 55: 06-19: 00: 00$ & 4.89 \\
\hline 46 & 22 Apr 2001 & $10: 04: 33-10: 06: 47$ & 2.17 \\
\hline 47 & 29 Apr 2001 & $13: 41: 12-13: 43: 22$ & 2.16 \\
\hline 48 & 2 May 2001 & $06: 23: 23-06: 26: 17$ & 2.89 \\
\hline
\end{tabular}

the $2-80 \mathrm{kHz}$ frequency range and the total signal energy, $E_{\text {pow }}$, calculated from the accumulated squared samples. The FFT technique is used to obtain the spectra. Both the elec- tric field power and spectra are available onboard each $13 \mathrm{~ms}$. However, due to telemetry limitations only averaged values of these parameters are transmitted on-ground. For the spectra, the time resolutions achieved are $2.15 \mathrm{~s}$ in the normal telemetry mode and $0.32 \mathrm{~s}$ in the burst mode. For $E_{\text {pow }}$ parameters, the corresponding time resolutions are $213 \mathrm{~ms}$ and $13 \mathrm{~ms}$, respectively (Décréau et al., 1997). Further details on different operational modes of WEC and WHISPER are described elsewhere (Pedersen et al., 1997; Décréau et al., 1997; Woolliscroft et al., 1997).

We chose 48 crossings of the foreshock boundary with dominating Langmuir waves and tried to find periodicities in the variations of their intensities.

From the results of numerical modeling performed by Winske and Quest (1988) it follows that the wavelength of wrinkles is $\sim 5 \mathrm{c} / \omega_{p i}$, with the frequency of rippling being $0.4 f_{B i}$. For the average solar wind magnetic field and density, $B=6 \mathrm{nT}$ and $N_{e}=6.5 \mathrm{~cm}^{-3}$, the proton gyrofrequency equals $\sim 0.1 \mathrm{~Hz}$, the proton gyroperiod is $11 \mathrm{~s}$, and the proton inertial scale is $c / \omega_{p i}=90 \mathrm{~km}$. Thus we expect that the characteristic temporal and spatial scales of the wrinkles are about $25 \mathrm{~s}$ (the corresponding frequency is $0.04 \mathrm{~Hz}$ ) and $500 \mathrm{~km}$, respectively. For the time period considered, the Cluster spacecraft separation is within the range 190$1700 \mathrm{~km}$, smaller or comparable to the wavelength of ripples observed in the numerical modeling by Winske and Quest (1988).

To detect periodicities that are possibly related to rippling and wrinkling, it is desirable to have time intervals containing more or about 4 rippling/wrinkling periods. On the other hand, the intervals should be short enough in order to avoid large changes of wave intensity due to approaching the foreshock boundary or moving away from it. Thus time intervals of 1.5-2 min seem to be optimal for the problem under consideration. The list of intervals with their durations is presented in Table 1.

For each event we estimated the corresponding proton gyrofrequency, solar wind Alfvén Mach number, as well as the proxies for shock Alfvén, sound, and fast Mach numbers. The proton density, plasma temperature, solar wind speed, and magnetic field, which were required for the estimation, were taken from the 1-min spacecraft-interspersed data set at $1 \mathrm{AU}$ (OMNI data set provided by CDAWeb facility) and averaged over time intervals corresponding to the events under study. For simplicity, the formulas for Alfvén and sound speeds assume a constant $4 \%$ alpha particle contribution and constant electron temperature, $T_{e}=1.28 \times 10^{5} \mathrm{~K}$. In particular, Alfvén and sound speeds are given by

$V_{A}=\frac{B}{\sqrt{\mu_{0}\left(4 n_{\alpha}+n_{p}\right) m_{p}}}$,

and

$V_{S}=\sqrt{\frac{2 k\left(T_{e}+T_{i}\right)}{\left(4 n_{\alpha}+n_{p}\right) m_{p}}}$, 


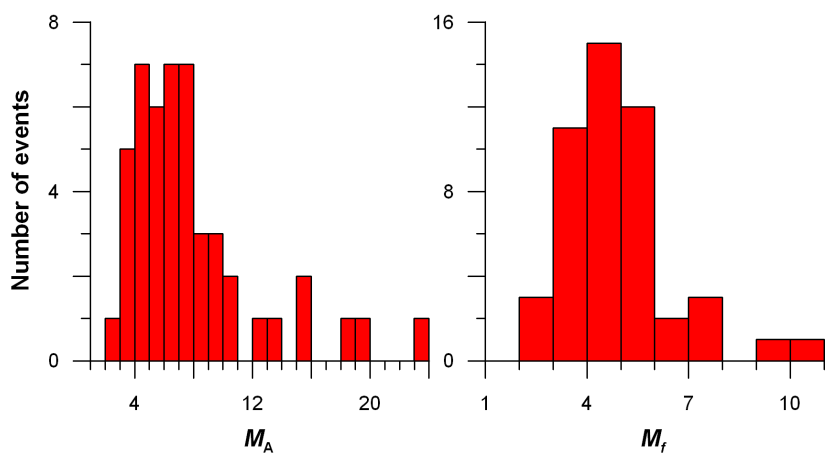

Fig. 1. Histograms of proxies for Alfvén and fast Mach numbers characterizing quasiperpendicular shocks related to the selected crossings of the foreshock boundary.

respectively ( $\mu_{0}$ is the permeability of free space and $k$ is the Boltzmann constant). The fast mode velocity is calculated for waves propagating at the right angle to the ambient magnetic field,

$V_{f}=\sqrt{V_{A}^{2}+V_{s}^{2}}$.

To calculate the proxies for the shock Mach numbers, the solar wind velocities were decomposed into two components, parallel and perpendicular to the solar wind magnetic field, and the perpendicular components were used. The obtained estimates of the solar wind Alfvén Mach numbers and the proxies of the shock Mach numbers are summarized in Table 2. It is worth noting that on average the proxies should overestimate the corresponding Mach numbers because we take projections of the plasma speed onto the magnetic field rather than onto the shock normal. Nevertheless a strong correlation should exist between the Mach numbers and such proxies, thereby allowing one to use the proxies in statistical studies. On the other hand, an accurate estimation of shock normals is rather difficult and their use would introduce additional errors, especially in the cases when the observations are performed far from the bow shock surface. For brevity, in the following discussion of experimental results we will speak about Mach numbers rather than their proxies.

From Table 2, it is clearly seen that the data set contains events related both to weak and strong shocks, with Alfvén and fast Mach numbers varying in the ranges 2.7-23.2 and 2.2-10.7, respectively. The histograms for Mach number proxies are shown in Fig. 1. It is worth noting that for the most of the events, 30 of 48, the Alfvén Mach number exceeds the value of 5.7 that was used in the simulations of Lowe and Burgess (2003). Fourteen events have an Alfvén Mach number higher than 8 , which is the value used by Winske and Quest (1988) in their simulations. For 6 events, the Alfvén Mach number exceeds 13, the value used in the simulations by Thomas (1989).

Because the measured wave energies $E_{\text {pow }}$ may vary over a broad range within the same event, the spectral analysis
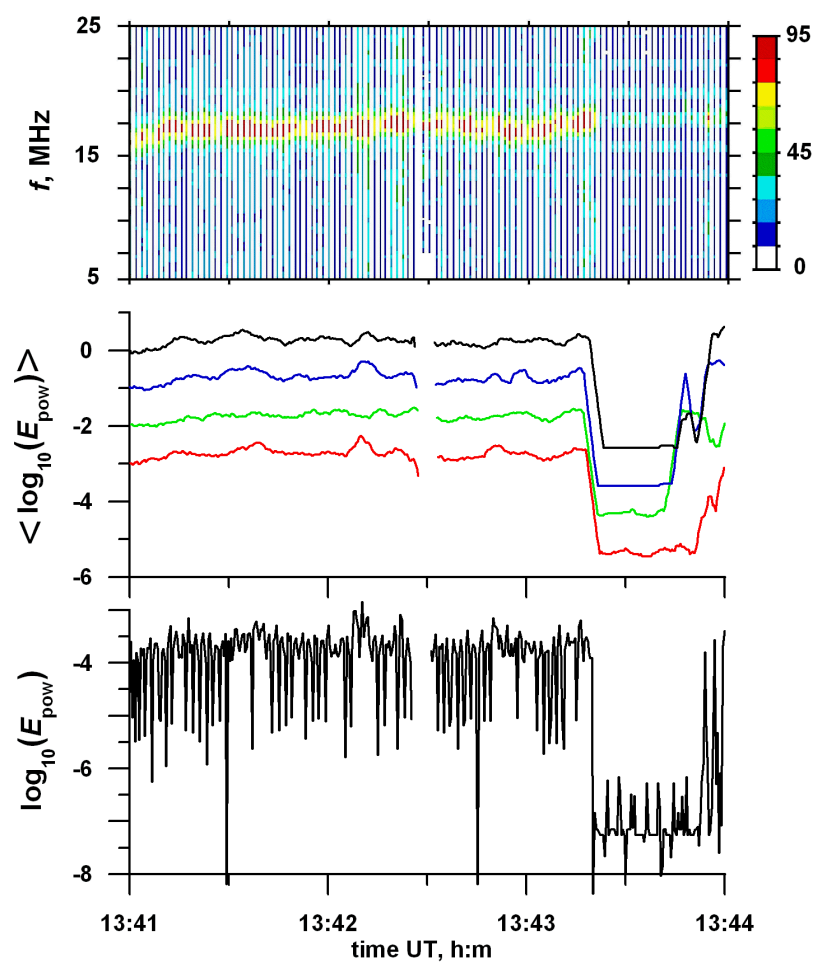

Fig. 2. (a) Frequency-time spectrograms obtained by the WHISPER instrument aboard Sc1 in the Earth's electron foreshock on 29 April 2001 (event 47). The electric field strength in $V_{\mathrm{rms} \mathrm{Hz}} \mathrm{Hz}^{-1 / 2}$ is color coded and plotted in $\mathrm{dB}$ over $10^{-7} V_{\mathrm{rms}} \mathrm{Hz}^{-1 / 2}$. The corresponding color scale is shown on the right of the spectrogram. (b) Profiles for $\log _{10}\left(E_{\text {pow }}\right)$ measured aboard all four spacecraft upon sliding averaging over $4 \mathrm{~s}$ time intervals. Red, green, blue, and black lines correspond to $\mathrm{Sc} 1, \mathrm{Sc} 2, \mathrm{Sc} 3$, and $\mathrm{Sc} 4$, respectively. To ease the comparison, the curves showing the measurements aboard $\mathrm{Sc} 2, \mathrm{Sc} 3$, and $\mathrm{Sc} 4$ are shifted upward by 1,2, and 3, respectively. (c) Profiles for $\log _{10}\left(E_{\text {pow }}\right)$ measured aboard Sc1. Strong modulation due to spacecraft spin is easily seen.

was performed for their $\log$ arithms, $\log _{10}\left(E_{\text {pow }}\right)$. Moreover, to avoid spectral artifacts due to spikes and trends, the temporal series of $\log _{10}\left(E_{\text {pow }}\right)$ were clipped at the levels of $\pm 3 \sigma$, where $\sigma$ is the standard deviation of $\log _{10}\left(E_{\text {pow }}\right)$, and linear trends were removed (if any).

All the events under consideration contain gaps (missing data). During the time intervals corresponding to the gaps, WHISPER worked in active (sounding) mode and the information about natural wave activity is not available. The duration of each gap is comparable to the period of spacecraft spin and much longer than the typical interval between two successive measurements of wave energies outside the gaps. It is well-known that different techniques of filling data gaps (e.g. by zeros or interpolated values) may work rather poorly and often result in spurious peaks corresponding to periods comparable to gaps. Fortunately, Lomb (1976) developed a method of spectral analysis without such shortcomings. 
Table 2. Parameters of the shocks related to the events studied.

\begin{tabular}{|c|c|c|c|c|c|}
\hline \multirow[b]{2}{*}{ No } & \multirow{2}{*}{$\begin{array}{r}\text { Solar } \\
\text { wind } \\
\text { Alfvén } \\
\text { Mach } \\
\text { number }\end{array}$} & \multicolumn{3}{|c|}{ Shock Mach number proxies } & \multirow{2}{*}{$\begin{array}{c}\text { Upstream proton } \\
\text { gyrofrequency, } \\
f_{B i}, \mathrm{~Hz}\end{array}$} \\
\hline & & Alfvén & sound & fast & \\
\hline 1 & 14.0 & 12.0 & 5.9 & 5.3 & 0.113 \\
\hline 2 & 6.7 & 4.9 & 5.0 & 3.5 & 0.171 \\
\hline 3 & 7.8 & 7.7 & 9.3 & 5.9 & 0.083 \\
\hline 4 & 6.8 & 6.6 & 8.6 & 5.2 & 0.102 \\
\hline 5 & 6.6 & 5.5 & 6.2 & 4.1 & 0.104 \\
\hline 6 & 6.8 & 5.7 & 6.2 & 4.2 & 0.076 \\
\hline 7 & 6.8 & 5.5 & 5.5 & 3.9 & 0.128 \\
\hline 8 & 15.6 & 15.6 & 7.0 & 6.4 & 0.061 \\
\hline 9 & 15.7 & 15.6 & 7.0 & 6.4 & 0.059 \\
\hline 10 & 8.4 & 8.3 & 6.6 & 5.2 & 0.094 \\
\hline 11 & 4.7 & 4.4 & 6.6 & 3.7 & 0.223 \\
\hline 12 & 6.8 & 6.4 & 6.1 & 4.4 & 0.172 \\
\hline 13 & 7.2 & 7.2 & 6.4 & 4.8 & 0.166 \\
\hline 14 & 8.7 & 5.5 & 4.2 & 3.3 & 0.143 \\
\hline 15 & 8.9 & 8.0 & 6.0 & 4.8 & 0.125 \\
\hline 16 & 9.2 & 9.1 & 7.4 & 5.7 & 0.059 \\
\hline 17 & 8.8 & 7.4 & 6.6 & 4.9 & 0.087 \\
\hline 18 & 9.3 & 9.1 & 7.5 & 5.8 & 0.082 \\
\hline 19 & 5.7 & 3.9 & 5.2 & 3.1 & 0.143 \\
\hline 20 & 6.7 & 6.2 & 6.3 & 4.4 & 0.101 \\
\hline 21 & 8.1 & 7.8 & 7.1 & 5.2 & 0.077 \\
\hline 22 & 7.4 & 4.4 & 3.6 & 2.8 & 0.074 \\
\hline 23 & 7.2 & 6.3 & 6.7 & 4.6 & 0.090 \\
\hline 24 & 7.2 & 7.1 & 6.7 & 4.9 & 0.083 \\
\hline 25 & 8.3 & 3.7 & 2.8 & 2.2 & 0.096 \\
\hline 26 & 20.8 & 19.4 & 7.7 & 7.2 & 0.037 \\
\hline 27 & 4.4 & 4.0 & 6.1 & 3.3 & 0.123 \\
\hline 28 & 5.1 & 4.2 & 4.9 & 3.2 & 0.109 \\
\hline 29 & 5.8 & 5.6 & 6.4 & 4.2 & 0.103 \\
\hline 30 & 10.3 & 10.0 & 6.6 & 5.5 & 0.074 \\
\hline 31 & 6.2 & 5.9 & 8.8 & 4.9 & 0.222 \\
\hline 32 & 3.8 & 3.8 & 10.2 & 3.6 & 0.214 \\
\hline 33 & 2.8 & 2.7 & 9.2 & 2.6 & 0.259 \\
\hline 34 & 6.4 & 6.3 & 6.0 & 4.3 & 0.136 \\
\hline 35 & 25.3 & 23.2 & 8.2 & 7.7 & 0.056 \\
\hline 36 & 10.0 & 8.9 & 7.2 & 5.6 & 0.170 \\
\hline 37 & 4.5 & 4.3 & 6.5 & 3.6 & 0.070 \\
\hline 38 & 4.8 & 4.7 & 10.2 & 4.2 & 0.092 \\
\hline 39 & 20.4 & 10.2 & 5.3 & 4.7 & 0.058 \\
\hline 40 & 18.9 & 18.9 & 13.0 & 10.7 & 0.053 \\
\hline 41 & 13.8 & 13.4 & 12.7 & 9.2 & 0.061 \\
\hline 42 & 8.2 & 6.9 & 7.9 & 5.2 & 0.103 \\
\hline 43 & 8.8 & 7.3 & 6.6 & 4.9 & 0.110 \\
\hline 44 & 8.6 & 3.7 & 6.0 & 3.2 & 0.065 \\
\hline 45 & 10.0 & 9.9 & 10.0 & 7.0 & 0.058 \\
\hline 46 & 3.9 & 3.8 & 8.1 & 3.5 & 0.226 \\
\hline 47 & 8.1 & 6.5 & 10.2 & 5.5 & 0.090 \\
\hline 48 & 7.3 & 7.2 & 8.3 & 5.4 & 0.103 \\
\hline
\end{tabular}

This method was based in part on earlier works by Barning (1963) and Vaníček (1971) and was elaborated further by Scargle (1982). They evaluate the spectra with the use of data that are actually measured, and, moreover, the measurements can be unevenly sampled. An implementation of Lomb's method used in the present paper is developed by Press et al. (1997). The maxima found in the Lomb normalized periodograms are considered as corresponding to statistically significant periodicities if their significance exceeds 0.05 . The significance is estimated against a hypothesis of random noise. Usually the spectra contain peaks in the vicinity of $0.5 \mathrm{~Hz}$ and their harmonics. These peaks result from rotation of spacecraft antennas with a period of $\sim 4 \mathrm{~s}$. They can be rather huge and bring down simple estimates of significance of other periodicities. Because of this, it is necessary to filter such time series in order to eliminate the oscillations resulting from spacecraft spin. The filtering is performed if the spin peaks are significant in accordance with the chosen criterion. To this end we use the harmonic filtering procedure which was developed by Ferraz-Mello (1981).

\section{Results and discussion}

An example of Langmuir waves associated with a low-Machnumber stationary shock is shown in Fig. 2. For this event the electron foreshock boundary was crossed at 13:43:22 UT, at the end of the time interval chosen for search of hidden periodicities. In addition to the frequency-time spectrogram obtained by the WHISPER instrument, shown are the profiles for $\log _{10}\left(E_{\text {pow }}\right)$ measured aboard all 4 spacecraft and averaged over $4 \mathrm{~s}$ time intervals equal to the satellite spin period, as well as unaveraged data for spacecraft $1(\mathrm{Sc} 1)$. From the frequency-time diagram it is easily seen that the wave activity is observed in a vicinity of local plasma frequency, both upshifted and downshifted oscillations are absent. Hence, the integrated signal energy can be attributed to Langmuir waves.

The results of spectral analysis obtained with the techniques outlined above are presented in Fig. 3. The periodograms of $\log _{10}\left(E_{\text {pow }}\right)$ for this event reveal a high and sharp peak at $f=0.5 \mathrm{~Hz}$, which is twice the spacecraft spin frequency. This modulation is also conspicuous in the unaveraged profile of $\log _{10}\left(E_{\text {pow }}\right)$ (Fig. 2, bottom panel). Such strong modulations of the wave energy are usually observed when the waves are linearly polarized and the angle between electric field vector and normal to the plane with antennas is not too small. Langmuir waves not far from their origin should be polarized approximately along the solar wind magnetic field, in the direction of propagation of energetic electrons reflected by the bow shock. In the frequency range of interest $\left(0.33 f_{B i} \leq f \leq f_{B i}\right)$ the observed variations of the wave energy is weak aboard all spacecraft but Sc3. However, the absence of significant peaks in the vicinity of the peak $f=0.69 f_{B i}$, which is found for Sc 3 , most probably indicates that this peak is spurious or caused by a local disturbance of the solar wind. Larger peaks exist for low frequencies outside of the range of interest $\left(0.33 f_{B i} \leq f \leq f_{B i}\right)$. These larger 

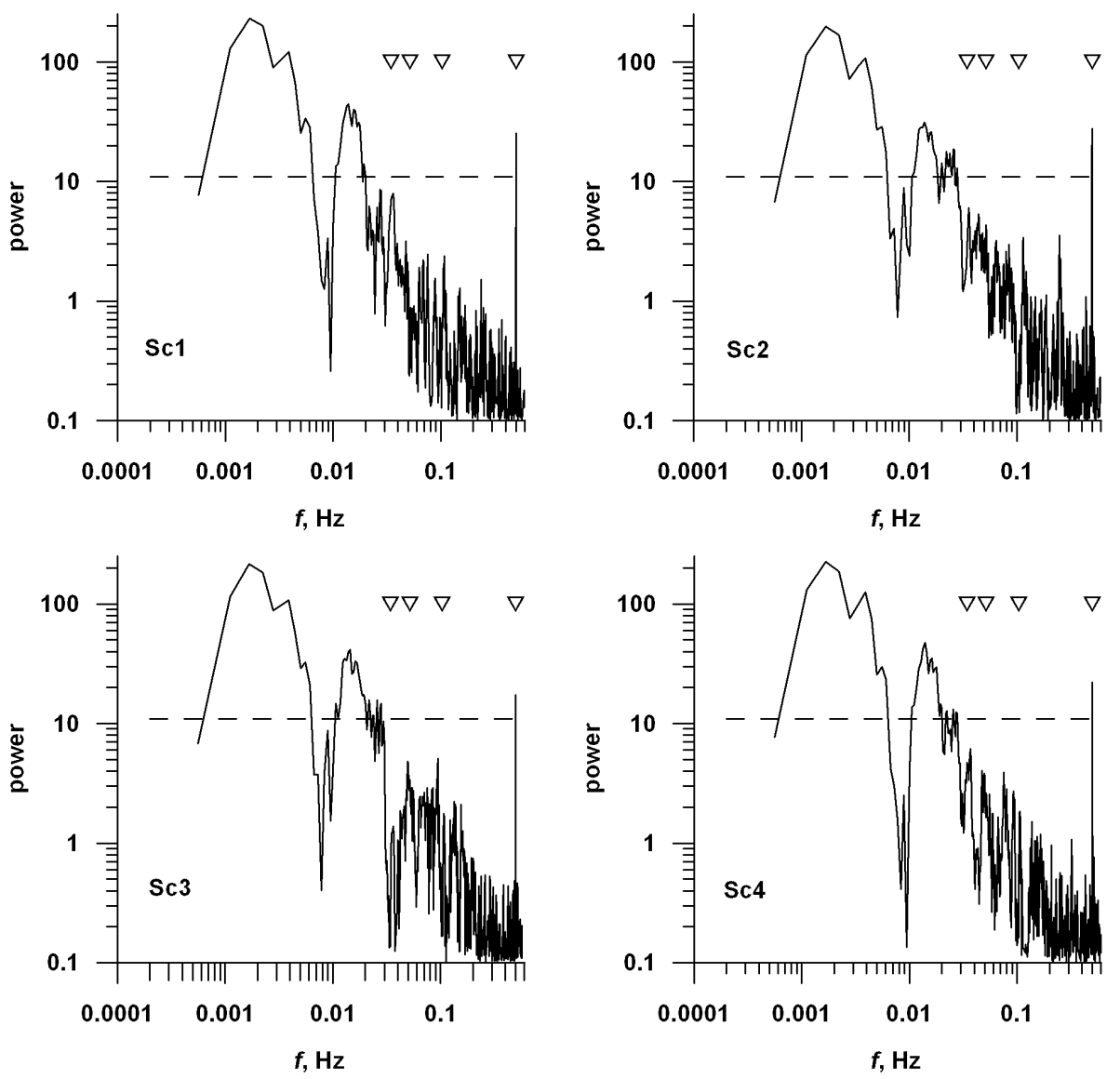

Fig. 3. Periodograms for wave energy densities measured at the foreshock boundary crossed by Cluster spacecraft on 29 April 2001 (event 47). Dashed lines correspond to the significance level of 0.05 . Abscissas of triangles correspond to $0.33 f_{B i}, 0.5 f_{B i}, f_{B i}$, and $0.5 \mathrm{~Hz}$. The frequency range of interest is located between the 1st and 3rd triangles, while the last one shows the position of peak resulting from spacecraft spin.

peaks may correspond to turbulent changes in the direction of the interplanetary magnetic field or the intrinsic burstiness of the Langmuir waves predicted by Stochastic Growth Theory (e.g. Robinson, 1995; Cairns and Robinson, 1999; Krasnoselskikh et al., 2007, and references therein).

The profiles for $\log _{10}\left(E_{\text {pow }}\right)$ measured at the boundary of the electron foreshock associated with a high-Mach-number shock (proxy for $M_{A}$ is equal to 10.2) are shown in Fig. 4, together with the periodogram for Sc1. Within the chosen time interval both upshifted and downshifted oscillations are absent, as in the previous case, and the integrated signal energy can be attributed to Langmuir waves. The periodogram contains a sharp peak at $f=0.5 \mathrm{~Hz}$ corresponding to effects of spacecraft spin. In the frequency range $0.33 f_{B i} \leq f \leq f_{B i}$ there is a high peak at $f=0.03 \mathrm{~Hz}\left(f / f_{B i}=0.55\right)$. Such peaks are also found both for Sc3 and Sc4, while for Sc2 the oscillations in this range are statistically insignificant. The results of statistical studies outlined in the following allow one to suggest that such oscillations are closely related to shock front nonstationarity. However, a more careful analysis, which is beyond the scope of the present paper, is necessary to confirm or reject such hypothesis for each particular event. Nevertheless this example can be considered as a quite typical event associated with a high-Mach-number shock. We observe that profiles for $\log _{10}\left(E_{\text {pow }}\right)$ measured aboard different spacecraft for this event do have similar features but also substantial differences. If we recall that the distances between spacecraft (510-730 km) are smaller or comparable to the expected spatial scale of rippling $(\sim 900 \mathrm{~km})$, it follows that such a rippling, if any, seems to be rather chaotic rather than regular, both in space and time. This conclusion is confirmed by examination of similar plots for all chosen events corresponding to high Mach numbers.

For all events listed in Table 1 we performed spectral analysis with the techniques outlined in the previous section. The parameters characterizing periodicities in the frequency range $0.33 f_{B i} \leq f \leq f_{B i}$ are presented in Table 3 . It is easily seen from Table 3 that there exists a lot of events with statistically significant periodicities. For several events the periodicities observed by different spacecraft are the same 
Table 3. Periodicities found in the wave energies.

\begin{tabular}{|c|c|c|c|c|c|c|c|c|c|c|c|c|}
\hline \multirow{3}{*}{$\begin{array}{c}\text { No } \\
1\end{array}$} & \multicolumn{12}{|c|}{ Frequency $f\left(10^{-2} \mathrm{~Hz}\right)$, ratio $f / f_{B i}$, and significance ${ }^{\mathrm{a}}$} \\
\hline & \multicolumn{4}{|c|}{ Sc1 } & \multicolumn{2}{|c|}{$\mathrm{Sc} 2$} & \multicolumn{3}{|c|}{$\mathrm{Sc} 3$} & \multicolumn{3}{|c|}{$\operatorname{Sc} 4$} \\
\hline & 5.4 & 0.47 & 1.7 & 6.3 & 0.55 & 3.5 & 6.0 & 0.53 & 3.0 & 5.7 & 0.50 & 3.3 \\
\hline 2 & & none & & & none & & & none & & & none & \\
\hline 3 & 3.5 & 0.42 & 3.2 & 4.1 & 0.49 & v 3.8 & 4.1 & 0.49 & 2.1 & 3.8 & 0.46 & 4.1 \\
\hline 4 & 4.1 & 0.40 & 3.2 & 4.4 & 0.43 & 8.7 & & none & & 4.5 & 0.44 & 3.3 \\
\hline 5 & 7.0 & 0.67 & 9.6 & 6.8 & 0.65 & 11.8 & 6.8 & 0.65 & 9.8 & 6.8 & 0.65 & 2.5 \\
\hline 6 & 4.2 & 0.55 & 9.3 & 4.2 & 0.55 & 13.9 & 4.9 & 0.64 & 8.5 & 4.9 & 0.64 & 5.0 \\
\hline 7 & 4.8 & 0.37 & 2.7 & & $\mathrm{n} / \mathrm{a}$ & & & $\mathrm{n} / \mathrm{a}$ & & & $\mathrm{n} / \mathrm{a}$ & \\
\hline 8 & 2.5 & 0.41 & 2.4 & 2.5 & 0.42 & 1.4 & 2.5 & 0.42 & 2.5 & 2.5 & 0.42 & 1.6 \\
\hline 9 & 3.0 & 0.51 & 5.2 & & none & & 3.0 & 0.51 & 2.6 & & none & \\
\hline 10 & 4.7 & 0.50 & 2.0 & 4.5 & 0.48 & 3.1 & 4.5 & 0.48 & 3.2 & 4.5 & 0.48 & 2.9 \\
\hline 11 & & none & & & none & & & none & & & none & \\
\hline 12 & & none & & & $\mathrm{n} / \mathrm{a}$ & & & none & & & none & \\
\hline 13 & & none & & & $\mathrm{n} / \mathrm{a}$ & & & none & & & none & \\
\hline 14 & & none & & & $\mathrm{n} / \mathrm{a}$ & & & none & & & $\mathrm{n} / \mathrm{a}$ & \\
\hline 15 & 5.9 & 0.47 & 1.6 & & $\mathrm{n} / \mathrm{a}$ & & 3.1 & 0.53 & 3.3 & & $\mathrm{n} / \mathrm{a}$ & \\
\hline 16 & & none & & & $\mathrm{n} / \mathrm{a}$ & & 4.7 & 0.81 & 5.1 & 4.3 & 0.73 & 3.5 \\
\hline 17 & 3.2 & 0.36 & 3.4 & 5.0 & 0.58 & 1.7 & & none & & & none & \\
\hline 18 & & none & & 6.9 & 0.84 & 1.5 & & none & & & none & \\
\hline 19 & & none & & & none & & & none & & & none & \\
\hline 20 & & none & & & none & & 6.5 & 0.64 & 1.4 & & $\mathrm{n} / \mathrm{a}$ & \\
\hline 21 & 3.2 & 0.42 & 5.0 & 3.6 & 0.48 & 4.0 & 3.8 & 0.50 & 15.3 & 3.2 & 0.42 & 2.7 \\
\hline 22 & 4.0 & 0.55 & 5.1 & 4.0 & 0.55 & 1.9 & 3.8 & 0.51 & 10.6 & 4.0 & 0.55 & 2.9 \\
\hline 23 & & none & & 3.6 & 0.48 & 4.0 & & none & & 8.3 & 0.92 & 2.0 \\
\hline 24 & 6.2 & 0.75 & 1.9 & 2.8 & 0.33 & 12.3 & 4.4 & 0.53 & 8.8 & 3.9 & 0.47 & 5.9 \\
\hline 25 & 8.7 & 0.91 & 1.9 & 3.8 & 0.40 & 1.5 & 8.8 & 0.92 & 4.6 & & $\mathrm{n} / \mathrm{a}$ & \\
\hline 26 & & none & & 2.6 & 0.69 & 2.6 & & none & & & none & \\
\hline 27 & 5.0 & 0.40 & 1.7 & 5.0 & 0.40 & 2.2 & & none & & 6.8 & 0.55 & 1.6 \\
\hline 28 & 5.8 & 0.53 & 2.3 & 5.7 & 0.52 & 1.9 & 4.5 & 0.41 & 2.4 & & $\mathrm{n} / \mathrm{a}$ & \\
\hline 29 & 4.4 & 0.43 & 7.1 & 4.2 & 0.41 & 6.5 & 4.2 & 0.41 & 6.8 & & $\mathrm{n} / \mathrm{a}$ & \\
\hline 30 & & $\mathrm{n} / \mathrm{a}$ & & 4.3 & 0.58 & 8.0 & 3.1 & 0.42 & 1.4 & & $\mathrm{n} / \mathrm{a}$ & \\
\hline 31 & & none & & & none & & & none & & & none & \\
\hline 32 & & none & & & none & & 8.9 & 0.41 & 2.7 & & none & \\
\hline 33 & & none & & & none & & & none & & & none & \\
\hline 34 & & none & & 8.0 & 0.65 & 1.8 & 7.3 & 0.59 & 2.0 & & none & \\
\hline 35 & 2.4 & 0.44 & 10.7 & 2.4 & 0.44 & 10.9 & 2.1 & 0.38 & 14.6 & 2.5 & 0.44 & 10.3 \\
\hline 36 & & none & & 5.9 & 0.61 & 3.0 & 5.9 & 0.61 & 4.1 & 6.2 & 0.64 & 7.4 \\
\hline 37 & & none & & & none & & & none & & & none & \\
\hline 38 & & none & & 8.7 & 0.48 & 1.9 & & none & & 6.6 & 0.36 & 1.4 \\
\hline 39 & 3.2 & 0.55 & 4.8 & & none & & 3.2 & 0.55 & 5.2 & 3.2 & 0.55 & 3.4 \\
\hline 40 & 2.2 & 0.42 & 3.7 & 1.9 & 0.35 & 10.2 & 2.3 & 0.43 & 5.6 & 2.4 & 0.46 & 2.4 \\
\hline 41 & 3.5 & 0.57 & 2.2 & 3.5 & 0.57 & 3.7 & 3.5 & 0.57 & 2.2 & 3.5 & 0.57 & 3.2 \\
\hline 42 & 4.5 & 0.43 & 5.2 & 4.7 & 0.45 & 9.1 & 5.1 & 0.49 & 5.1 & 5.3 & 0.51 & 2.9 \\
\hline 43 & 6.0 & 0.54 & 3.1 & 5.2 & 0.47 & 2.0 & 4.9 & 0.45 & 3.3 & 4.9 & 0.45 & 2.9 \\
\hline 44 & & none & & 5.7 & 0.88 & 2.7 & & $\mathrm{n} / \mathrm{a}$ & & 5.7 & 0.88 & 1.8 \\
\hline 45 & & none & & 2.5 & 0.43 & 2.0 & & $\mathrm{n} / \mathrm{a}$ & & & $\mathrm{n} / \mathrm{a}$ & \\
\hline 46 & & none & & & none & & & none & & & none & \\
\hline 47 & & none & & & none & & 6.2 & 0.69 & 3.8 & & none & \\
\hline 48 & & none & & & none & & & none & & & none & \\
\hline
\end{tabular}

The abbreviation "n/a" stands for absent data, while "none" means that no significant periodicity is found in the frequency range of interest. 

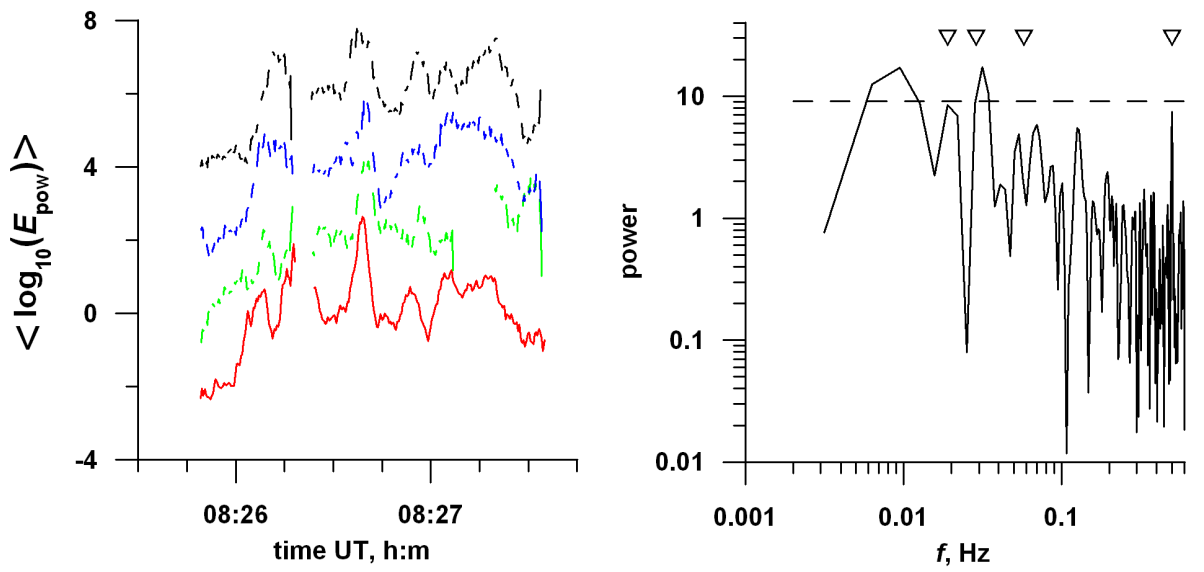

Fig. 4. (a) Profiles for $\log _{10}\left(E_{\text {pow }}\right)$ measured aboard all four Cluster spacecraft upon sliding averaging over $4 \mathrm{~s}$ time intervals. Measurements were performed by the WHISPER instrument in the Earth's electron foreshock on 29 March 2001 (event 39). Red, green, blue, and black lines correspond to $\mathrm{Sc} 1, \mathrm{Sc} 2, \mathrm{Sc} 3$, and Sc4, respectively. To ease the comparison, the curves showing the measurements aboard Sc2, Sc3, and Sc4 are shifted upward by 2, 4, and 6, respectively. (b) Periodogram for wave energy density measured aboard Sc1. The dashed line shows the significance level of 0.05 . The triangles have abscissas at $0.33 f_{B i}, 0.5 f_{B i}, f_{B i}$, and $0.5 \mathrm{~Hz}$. The frequency range of interest is located between the 1 st and 3rd triangles, while the last one shows the position of peak resulting from spacecraft spin.

or similar, while there is a number of events with different periodicities.

Obviously the observed oscillations of Langmuir wave intensities can be attributed both to the dynamics of the shock front itself and to some other phenomena that may be not directly related to the bow shock, e.g. to modulation of plasma and/or electron beam parameters by a MHD wave that was generated elsewhere and encountered the electron foreshock or the bow shock. Generally speaking, for any particular event a periodicity found cannot confidently be ascribed to shock front dynamics, first of all due to unavailability of simultaneous measurements in the vicinity of the shock front, where the energetic electrons are produced, and at the foreshock boundary where intense Langmuir waves are observed.

As usual when one has to deal with a number of unmonitored factors, a statistical approach is required. In particular, for oscillations resulting from shock front dynamics one can expect a correlation between shock Mach number and the probability of observing such oscillations. On the other hand, quite reliable estimates of shock Mach numbers associated with given observations at the foreshock boundaries cannot be obtained in the most of cases due to a number of reasons. However, a proxy for the Alfvén Mach number can easily be calculated from available data set by taking a projection of solar wind velocity onto the magnetic field at $1 \mathrm{AU}$, as described in Sect. 2.

We analyzed the dependence of the fractions of stationary and nonstationary shocks on the proxy for the shock Alfvén Mach number and on the solar wind Alfvén Mach number itself. The results obtained are shown in Fig. 5. Since the number of events is not very large, especially for small and large Mach numbers, for the points shown on the plots the

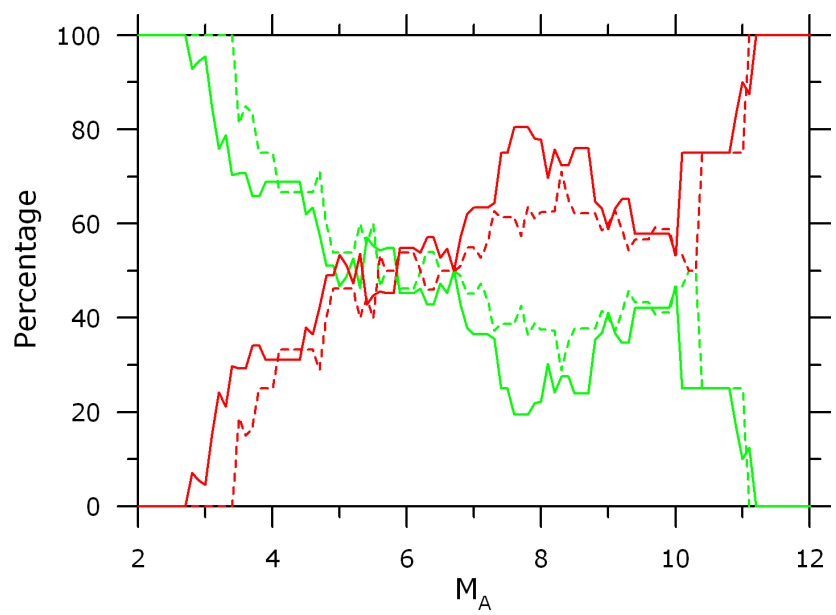

Fig. 5. Dependence of percentage of stationary (green lines) and nonstationary (red lines) shocks on the proxy of perpendicular Alfvén Mach number (solid lines) and on the solar wind Alfvén Mach number (dashed lines). To decrease statistical scattering, the number of shocks is calculated for Mach number intervals of length 2 for each point shown on the plots.

numbers of shocks are calculated for Mach number intervals of length 2 , with the abscissas of the points corresponding to the centers of the intervals. It follows from the plots that the probability for the shock to be stationary in this frequency range decreases as the Alfvén Mach number increases, as would be expected provided that there exists a strong correlation between the shock Alfvén Mach number and its proxy. Moreover, the probability to observe nonstationary shocks vanishes for low Mach numbers and approaches a unity as 
the Mach number increases up to high values, in accordance with the expectations based on theory, numerical simulations, and previous observations (e.g. Krasnoselskikh et al., 2002; Hellinger, 2003; Lembege et al., 2004; Bale et al., 2005). As should be expected, similar results are obtained with the solar wind Alfvén Mach numbers (see Fig. 5), most probably because solar wind and shock Mach numbers are highly correlated.

Analogous plots for dependencies on "parallel" Mach number proxy, which is calculated with the use of projection of plasma velocity onto the magnetic field, don't reveal a correlation between the probabilities and Mach number (the plots are not shown). This fact can be considered as additional evidence that the choice of Alfvén Mach number proxy is reasonable and the periodicities for the most of the events are related to intrinsic shock front dynamics rather than to external disturbances. Analogous plots with the sound and fast Mach number proxies reveal the same tendencies (the plots are not shown). However, the correlation between the probability for the shock to be nonstationary and these proxies are considerably lower than that for Alfvén Mach number. Most probably the main reasons for this are weaker correlations between these Mach numbers and their proxies. Indeed, in addition to solar wind velocity, density, and magnetic field required for calculation of Alfvén Mach number, the estimation of sound and fast Mach numbers needs additional information about electron and ion temperatures. The OMNI data contain only proton temperature, while the electron temperature is assumed to be the same for all events. In addition, the estimates of temperatures of different solar wind components usually have rather large uncertainties.

\section{Summary and conclusions}

In the present paper, we describe in detail and use the method for remote sensing of the quasiperpendicular part of the bow shock surface. This method was suggested by Lobzin et al. (2003) and was previously proven to be useful in a case study of a nonstationary bow shock observed by Cluster spacecraft on 24 January 2001 at 07:05:00-07:09:00 UT. The method is based on analysis of high frequency electric field fluctuations corresponding to Langmuir, upshifted, and downshifted oscillations in the electron foreshock. Langmuir waves usually have maximum intensity at the upstream boundary of this region. All these waves are commonly believed to be generated by energetic electrons accelerated at the quasiperpendicular zone of the shock front. Nonstationary behavior of the shock, in particular due to rippling, should result in modulation of energetic electron fluxes, thereby giving rise to variations of Langmuir waves intensity. For upshifted and downshifted oscillations, the variations of both intensity and central frequency can be observed. For the present study, WHISPER measurements of electric field spectra obtained aboard Cluster spacecraft are used to choose
48 crossings of the electron foreshock boundary with dominating Langmuir waves and to perform for the first time a statistical analysis of nonstationary behavior of quasiperpendicular zone of the Earth's bow shock. Analysis of hidden periodicities in plasma wave energy reveals shock front nonstationarity in the frequency range $0.33 f_{B i}<f<f_{B i}$, where $f_{B i}$ is the proton gyrofrequency upstream of the shock, and shows that the probability to observe such a nonstationarity increases with Mach number. The profiles observed aboard different spacecraft and the dominating frequencies of the periodicities are usually different. Hence nonstationarity and/or rippling seem to be rather irregular both in space and time rather than resembling a quasiregular wave-like pattern propagating on the shock surface. However, a possibility that effects of quasiregular oscillations of the shock may be obscured by the fluctuations of the solar wind parameters and due to other reasons cannot be definitely excluded.

Acknowledgements. The authors are thankful to Iver Cairns for useful discussions. This work was partially supported by le Studium program of Region Centre of France and by the Australian Research Council. The work of K. Musatenko was supported by the French Government Fellowship. This work was partially performed in the frame of ISSI team activities entitled "High-Mach-number collisionless shock dynamics: theory and simulations versus multipoint measurements in space" and it was also financially supported by CNES as Scientific Space Research Proposal entitled "Cluster Co-I DWP."

Topical Editor R. Nakamura thanks two anonymous referees for their help in evaluating this paper.

\section{References}

Bagenal, F., Belcher, J. W., Sittler Jr., E. C., and Lepping, R. P.: The Uranian bow shock: Voyager 2 inbound observations of a high Mach number shock, J. Geophys. Res., 92, 8603-8612, 1987.

Bale, S. D., Balikhin, M. A., Horbury, T. S., Krasnoselskikh, V. V., Kucharek, H., Möbius, E., Walker, S. N., Balogh, A., Burgess, D., Lembege, B., Lucek, E. A., Scholer, M., Schwartz, S. J., and Thomsen, M. F.: Quasi-perpendicular shock structure and processes, Space Sci. Rev., 118, 161-203, 2005.

Balikhin, M. A., Walker, S. N., de Wit, T. Dudok, Alleyne, H. St. C. K., Woolliscroft, L. J. C., MierJedrzejowicz, W. A. C., Baumjohann, W., and Barning, F. J. M., Non-stationarity and low frequency turbulence at a quasiperpendicular shock front, Adv. Space Res., 20, 729-734, 1997.

Barning, F. J. M.: The numerical analysis of the light-curve of 12 Lacertae, Bulletin of the Astronomical Institutes of the Netherlands, 17, 22-28, 1963.

Biskamp, D. and Welter, H.: Numerical studies of magnetosonic collisionless shock waves, Nucl. Fusion, 12, 663-666, 1972.

Burgess, D.: Interpreting multipoint observations of substructure at the quasi-perpendicular bow shock: Simulations, J. Geophys. Res., 111, A10210, doi:10.1029/2006JA011691, 2006.

Burgess, D. and Scholer, M.: Shock front instability associated with reflected ions at the perpendicular shock, Phys. Plasmas, 14, 012108, doi:10.1063/1.2435317, 2007. 
Cairns, I. H. and Robinson, P. A. Strong evidence for stochastic growth of Langmuir-like waves in Earth's foreshock, Phys. Rev. Lett., 82, 3066-3069, 1999.

Décréau, P. M. E., Fergeau, P., Krasnosel'skikh, V., Lévêque, M. L, Martin, Ph., Randriamboarison, O., Sené, F. X., Trotignon, J. G., Canu, P., Mögensen, P. B., and Whisper investigators: Whisper, a resonance sounder and wave analyser: performances and perspectives for the Cluster mission, Space Sci. Rev., 79, 157-193, 1997.

Ferraz-Mello, S.: Estimation of periods from unequally spaced observations, Astron. J., 86, 619-624, 1981.

Filbert, P. C. and Kellogg, P. J.: Electrostatic noise at the plasma frequency beyond the bow shock, J. Geophys. Res., 84, 1369$1381,1979$.

Galeev, A. A., Kennel, C. F., Krasnoselskikh, V. V., and Lobzin, V. V.: The role of whistler oscillations in the formation of the structure of high Mach number collisionless shock, in: Proceedings of the Joint Varenna-Abastumani International School and Workshop on Plasma Astrophysics, Varenna, Italy, 24 August3 September 1988, European Space Agency, Paris, 1988. ESA SP-285, vol. 1, pp. 165-171, 1988a.

Galeev, A. A., Kennel, C. F., Krasnoselskikh, V. V., and Lobzin, V. V.: Quasi-perpendicular collisionless high Mach number shocks, in: Proceedings of the Joint Varenna-Abastumani International School and Workshop on Plasma Astrophysics, Varenna, Italy, 24 August-3 September 1988, European Space Agency, Paris, 1988. ESA SP-285, vol. 1, pp. 173-183, 1988b.

Hellinger, P.: Structure and stationarity of quasi-perpendicular shocks: numerical simulations, Planet. Space Sci., 51, 649-657, 2003.

Horbury, T. S., Cargill, P. J., Lucek, E. A., Balogh, A., Dunlop, M. W., Oddy, T. M., Carr, C., Brown, P., Szabo, A., and Fornaçon, K.-H.: Cluster magnetic field observations of the bowshock: orientation, motion and structure, Ann. Geophys., 19, 1399-1409, 2001, http://www.ann-geophys.net/19/1399/2001/.

Kennel, C. F., Edmiston, J. P., and Hada, T.: A quarter century of collisionless shock research, in: Collisionless Shocks in the Heliosphere: A Tutorial Review, edited by: Stone, R. G. and Tsurutani, B. T., AGU, Washington, D.C., pp. 1-36, 1985.

Krasnoselskikh, V. V., Lobzin, V. V., and Rozov, V. B.: Nonstationary processes in the front of high Mach number collisionless quasiperpendicular shock: Dynamics of the ion distribution, in: Proceedings of the Joint Varenna-AbastumaniESA-Nagoya-Potsdam International Workshop on Plasma Astrophysics, Telavi, Georgia, 4-12 June 1990. European Space Agency, Paris, 1990, ESA SP-311, pp. 103-107, 1990.

Krasnosel'skikh, V. V., Balikhin, M. A., Alleyne, H. St. C., Klimov, S. I., Mier-Jedrzejowicz, W. A. C., Pardaens, A. K., Petrukovich, A., Southwood, D. J., Vinogradova, T., and Woolliscroft, L. J. C.: On the nature of low frequency turbulence in the foot of strong quasi-perpendicular shocks, Adv. Space Res., 11(9), 1518, 1991.

Krasnoselskikh, V. V., Lembege, B., Savoini, P., and Lobzin, V. V.: Nonstationarity of strong collisionless quasiperpendicular shocks: theory and full particle numerical simulations, Phys. Plasmas, 9, 1192-1209, 2002.

Krasnoselskikh, V. V., Lobzin, V. V., Musatenko, K., Soucek, J., Pickett, J. S., and Cairns, I. H.: Beam-plasma inter- action in randomly inhomogeneous plasmas and statistical properties of small-amplitude Langmuir waves in the solar wind and electron foreshock, J. Geophys. Res., 112, A10109, doi:10.1029/2006JA012212, 2007.

Lacombe, C., Mangeney, A., Harvey, C. C., and Scudder, J. D.: Electron plasma waves upstream of the Earth's bow shock, J. Geophys. Res., 90, 73-94, 1985.

Lembege, B., Giacalone, J., Scholer, M., Hada, T., Hoshino, M. Krasnoselskikh, V., Kucharek, H., Savoini, P., and Terasawa, T.: Selected problems in collisionless-shock physics, Space Sci. Rev., 110, 161-226, 2004.

Leroy, M. M. and Mangeney, A.: A theory of energization of solar wind electrons by the Earth's bow shock, Ann. Geophys., 2, 449456, 1984, http://www.ann-geophys.net/2/449/1984/.

Lobzin, V. V., Krasnoselskikh, V. V., Bosqued, J.-M., Pinçon, J.-L., Schwartz, S. J., and Dunlop, M.: Nonstationarity and reformation of high-Mach-number quasiperpendicular shocks: Cluster observations, Geophys. Res. Lett., 34, L05107, doi:10.1029/2006GL029095, 2007.

Lobzin, V. V., Décréau, P., Lefebvre, B., Krasnoselskikh, V., and Schwartz, S. J.: The Earth's bow shock nonstationarity evidenced by electrostastic waves within the electron foreshock, in: SpatioTemporal Analysis and Multipoint Measurements in Space, Orleans, France, 12-16 May 2003, pp. 44-45, 2003.

Lobzin, V. V., Krasnoselskikh, V. V., Schwartz, S. J., Cairns, I., Lefebvre, B., Décréau, P., and Fazakerley, A.: Generation of downshifted oscillations in the electron foreshock: a loss-cone instability, Geophys. Res. Lett., 32(18), L18101, doi:10.1029/2005GL023563, 2005.

Lomb, N. R.: Least-squares frequency analysis of unequally spaced data, Astrophys. Space Sci., 39, 447-462, 1976.

Lowe, R. E. and Burgess, D.: The properties and causes of rippling in quasi-perpendicular collisionless shock fronts, Ann. Geophys. 21, 671-679, 2003, http://www.ann-geophys.net/21/671/2003/.

Morse, D. L., Destler, W. W., and Auer, P. L.: Nonstationary behavior of collisionless shocks, Phys. Rev. Lett., 28, 13-16, 1972.

Moullard O., Burgess, D., Horbury, T. S., and Lucek, E. A.: Ripples observed on the surface of the Earth's quasiperpendicular bow shock, J. Geophys. Res., 111, A09113, doi:10.1029/2005JA011594, 2006

Pedersen, A., Cornilleau-Wehrlin, N., de La Porte, B., Roux, A., Bouabdellah, A., Decreau, P. M. E., Lefeuvre, F., Sène, F. X., Gurnett, D., Huff, R., Gustafsson, G., Holmgren, G., Woolliscroft, L., Alleyne, H. St. C., Thompson, J. A., and Davies, P. H. N.: The Wave Experiment Consortium (WEC), Space Sci. Rev., 79, 93-105, 1997.

Press, W.H., Teukolsky, S. A., Vetterling, W. T., Flannery, B. P.: Numerical Recipes in Fortran 77, The Art of Scientific Computing, 2nd Edition, vol. 1 of Fortran Numerical Recipes, Press Syndicate of the University of Cambridge, 1997.

Robinson, P. A.: Stochastic wave growth, Phys. Plasmas, 2, 1466 1479, 1995.

Scargle, J. D.: Studies in astronomical time series analysis. II. Statistical aspects of spectral analysis of unevenly spaced data, Astrophys. J., 263, 835-853, 1982.

Thomas, V. A.: Dimensionality effects in hybrid simulations of high Mach number collisionless perpendicular shocks, J. Geo- 
phys. Res., 94, 12 009-12 014, 1989.

Vaisberg, O., Klimov, S., Zastenker, G., Nozdratchev, M., Sokolov, A., Smirnov, V., Savin, S., and Avanov, L.: Relaxation of plasma at the shock front, Adv. Space Res., 4, 265-275, 1984.

Vaníček, P.: Further development and properties of the spectral analysis by least-squares, Astrophys. Space Sci., 12, 10-33, 1971.

Walker, S. N., Balikhin, M. A., and Nozdrachev, M. N.: Ramp nonstationarity and the generation of whistler waves upstream of a strong quasiperpendicular shock, Geophys. Res. Lett., 26, 13571360, 1999a.

Walker, S. N., Balikhin, M. A., Alleyne, H. St. C. K., Baumjohann, W., and Dunlop, M.: Observations of a very thin shock, Adv. Space Res., 24, 47-50, 1999b.
Winske, D. and Quest, K. B.: Magnetic field and density fluctuations at perpendicular supercritical collisionless shocks, J. Geophys. Res., 93, 9681-9693, 1988.

Woolliscroft, L. J. C., Alleyne, H. St. C., Dunford, C. M., Sumner, A., Thompson, J. A., Walker, S. N., Yearby, K. H., Buckley, A., Chapman, S., Gough, M. P., and the DWP Co-Investigators: The Digital-Wave-Processing experiment on Cluster, Space Sci. Rev., 79, 209-231, 1997.

Wu, C. S.: A fast Fermi process: energetic electrons accelerated by a nearly perpendicular bow shock, J. Geophys. Res., 89, 88578862, 1984.

Yuan, X., Cairns, I. H., and Robinson, P. A.: Simulation of energetic electron bursts upstream of re-forming shocks, Astrophys. J., 671, 439-446, 2007. 